\title{
Fluorescence optosensor using an artificial neural network for screening of polycyclic aromatic hydrocarbons
}

\author{
J.F. Fernández-Sánchez a ${ }^{\text {, A. Segura Carretero }}{ }^{\mathrm{a}, *}$, J.M. Benítez-Sánchez $^{\mathrm{b}}$, \\ C. Cruces-Blanco ${ }^{\mathrm{a}}$, A. Fernández-Gutiérrez ${ }^{\mathrm{a}}$ \\ ${ }^{a}$ Department of Analytical Chemistry, Faculty of Sciences, University of Granada, C/Fuentenueva s/ $n$, 18071 Granada, Spain \\ ${ }^{\mathrm{b}}$ Department of Computer Science and Artificial Intelligence, University of Granada, 18071 Granada, Spain
}

Received 1 May 2003; received in revised form 6 January 2004; accepted 13 January 2004

\begin{abstract}
This paper presents an optosensor for screening of four polycyclic aromatic hydrocarbons: anthracene (ANT), benzo[a]pyrene (BaP), fluoranthene (FLT), and benzo[b]fluoranthene (Bbf) using a photomultiplier device with an artificial neural network as transducer. The optosensor is based on the on-line immobilization on a non-ionic resin (Amberlite XAD-4) solid support in a continuous flow. The determination was performed in $15 \mathrm{mM} \mathrm{H}_{2} \mathrm{PO}_{4}{ }^{-} / \mathrm{HPO}_{4}{ }^{2-}$ buffer solution at $\mathrm{pH} 7$ and $25 \%$ of 1,4-dioxane. Feed forward neural networks (multiplayer perceptron) have been trained to quantify the considered Polycyclic aromatic hydrocarbons (PAHs) in mixtures under optimal conditions. The optosensor proposed was also applied satisfactorily to the determination of the considered PAHs in water samples in presence of the other 12 EPA-PAHs. (C) 2004 Elsevier B.V. All rights reserved.
\end{abstract}

Keywords: Fluorescence optosensor; Artificial neural networks; Screening; Polycyclic aromatic hydrocarbons; Water

\section{Introduction}

During the last 10 years, the advantages of using optosensors in analytical chemistry have been demonstrated [1,2]. Most of the optical optosensors developed so far have been designed for monitoring only one analyte and in just one position because a conventional spectroluminometer equipped with a photomultiplier (PMT) detector has been used as transducer [3].

However, in many situations (to carry out a screening method) it is required to perform the monitoring of several analytes simultaneously. In this case, it is necessary to use multichannel detectors such as Charge-Coupled Devices detectors (CCD), imaging techniques, chemometric models such as Partial Least Square (PLS), Principal Component Regression (PCR), Multiple Linear Regression (MLR) etc. or Artificial Neural Networks (ANN's). The multichannel devices or imaging techniques are more difficult to implement in a portable device and increase its price with respect to the PMT detectors. The chemometric models are easy to apply to solve mixtures of compound but it has been

\footnotetext{
* Corresponding author. Tel.: +34-958248593; fax: +34-958249510.

E-mail address: ansegura@ugr.es (A. Segura Carretero).
}

demonstrated over the last ten years than ANN's provide better results [4-8] and are more powerful tools for the analysis of mixtures with more than two components [9]. The main advantage of ANN's over the traditional non-linear regression techniques is the fact that they are able to generate models without a priory knowledge about the modelling function [10].

By coupling a PMT with ANN, it is possible to transform a singlechannel device in a multichannel detector, obtaining the properties of the PMTs (low cost, easy to further miniaturization) and the properties of the CCD's (simultaneous determinations).

A detailed description of the theory behind ANN can be found in the literature [11-21]. ANN's have been used for the processing of spectral data and, consequently, to determine simultaneously mixtures of several compounds [16]. Among the vast number of neural models proposed the most widely used is the multilayered perceptron (MLP), that is the type of ANN considered in this paper.

Polycyclic aromatic hydrocarbons (PAHs) are highly carcinogenic [22]. Identification and quantification of complex mixtures of PAHs need separation techniques as HPLC with UV-visible, fluorimetric or amperometric detection or GC-MS or GC-FID [23-27], and most of them include a 
preconcentration step. The combination of optosensor with ANN data processing is a good tool to solve this problem.

In this paper, a new optosensor for screening of four PAHs: anthracene (ANT), fluoranthene (FLT), benzo[a]pyrene $(\mathrm{BaP})$ and benzo[b]fluoranthene $(\mathrm{BbF})$ using a PMT as transducer with a MLP using the QuickProp training algorithm is presented. This optosensor diminishes the price of the sophisticated instrumentation and helps to miniaturize the system.

\section{Experimental}

\subsection{Chemicals and materials}

Analytical reagent grade chemicals were used for the preparation of all the solutions. Sodium di-hydrogen phosphate 1-hydrate was bought from Sigma (Spain) and used as received. A solution of $0.1 \mathrm{M} \mathrm{H}_{2} \mathrm{PO}_{4}{ }^{-} / \mathrm{HPO}_{4}{ }^{2-}$ buffer at pH 7.0 was prepared.

Fifty microgram per milliliter solutions of PAHs (Sigma) were prepared in 1,4-dioxane and kept at $4{ }^{\circ} \mathrm{C}$. The PAHs were handled with extreme caution; nitrile gloves were worn when working with the solutions and a low-maintenance respirator with the appropriate filters and disposable spatulas and weighing dishes when handling them in solid form. The wastes were collected in suitable residue containers for processing according to international norms.

The non-ionic resins Amberlite XAD 4 (Sigma) were sieved and used at $80-120 \mu \mathrm{m}$ size.

Water was distilled twice with a Mili-Q System (Millipore, Bedford, MA, USA).

\subsection{Optosensing manifold}

A $25 \mu \mathrm{l}$ Hellma 176.052-QS flow-through cell was packed with Amberlite XAD 4 and placed in the conventional sample compartment of the detector in a single-line flow-injection system (Fig. 1). Two rotary valves (A and B) (Supelco 5020) were used to introduce the sample and regenerate the active surface. PTFE tubing $(0.8 \mathrm{~mm}$ i.d.) and fittings were used to connect the flow-through cell. A Gilson Minipuls-3 peristaltic pump was used to generate the flow stream.

The fluorescence measurements were carried out with an Aminco Bowman Series 2 luminescence spectrometer fitted with a $150 \mathrm{~W}$ continuous high-power xenon lamp, two monochromators with a resolution of $0.2 \mathrm{~nm}$ and a high performance R928 PMT detector.

\subsection{Data analysis procedure}

The data was obtained by using a luminiscence spectometer. This instrument rendered the measured spectra in an ASCII file. This file was automatically transformed into a spreadhseet. To make easier the learning task of the ANN's some preliminary transformations were applied to the data.

To begin with, data were scaled down from their original domain to the unit interval $[0,1]$. Then a feature selection was applied. Not all the data gathered by the experimental equipment is relevant for the identification task. Experts' advice suggested to use only the spectra registered at a small set of wavelengths, namely, seven. The data for the learning of ANN consisted of only seven inputs and four outputs. The inputs are the fluorescence intensities and the outputs correspond to the percentage of concentration of each PAH, which are also scaled to $[0,1]$.

\subsection{General Procedure}

Samples were prepared by adding to the sample up to $25 \%$ (v/v) 1,4-dioxane and $15 \mathrm{mM} \mathrm{H}_{2} \mathrm{PO}_{4}{ }^{-} / \mathrm{HPO}_{4}{ }^{2-}$ buffer solution at $\mathrm{pH} 7.0 .5 \mathrm{ml}$ of the sample was injected through valve $A$ into a channel of $15 \mathrm{mM} \mathrm{H}_{2} \mathrm{PO}_{4}{ }^{-} / \mathrm{HPO}_{4}{ }^{2-}$ buffer solution at $\mathrm{pH}$ 7. The PAHs were kept in the flow cell in this medium at a $2.0 \mathrm{ml} \mathrm{min}^{-1}$ flow-rate on Amberlite XAD 4.

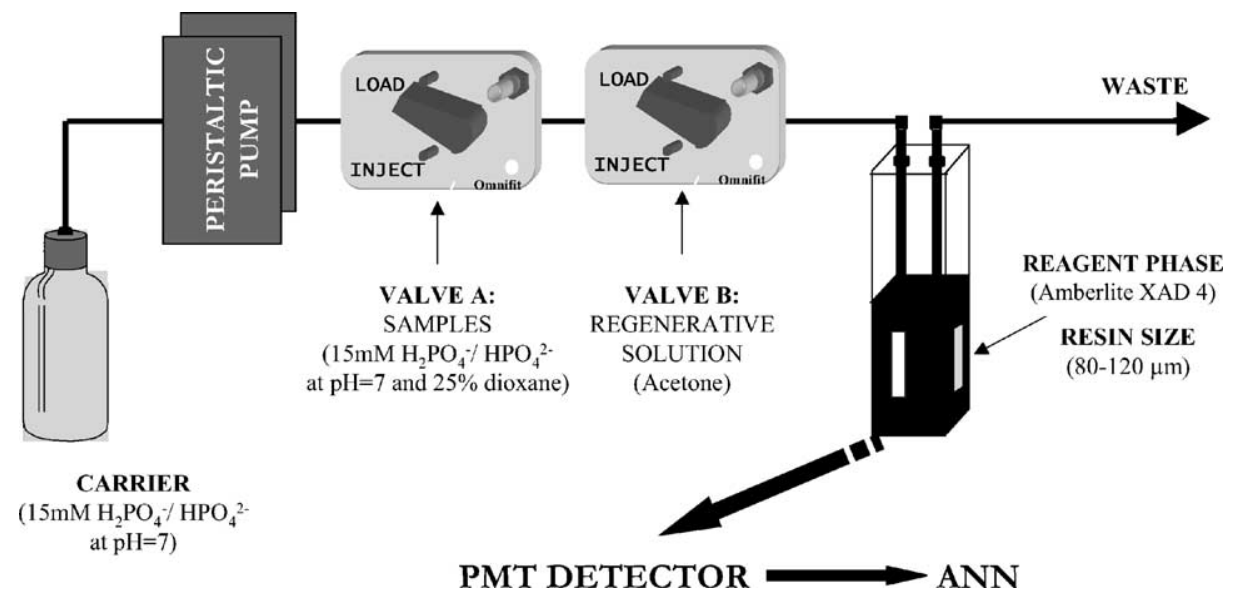

Fig. 1. Flow manifold used for fluorescence optosensor. (A) sample valve, and (B) regenerative solution valve. 
Emission spectra between $370-550 \mathrm{~nm}$, at scan rate of $3 \mathrm{~nm} \mathrm{~s}^{-1}$ and step size of $2 \mathrm{~nm}$, were carried out with an excitation wavelength of $355 \mathrm{~nm}$, detector voltage of $600 \mathrm{~V}$ and bandwidth slits of $4 \mathrm{~nm}$, saved as ASCII format file and processed following the Data Analysis Procedure.

Two hundred and fifty microliter of regenerative solution (acetone) was injected through valve B to strip the retained analyte from the reagent phase before proceeding with the next sample.

\section{Results and discussion}

\subsection{Chemical parameters}

Our research group have developed an optosensor for screening of four PAHs (ANT, BaP, FLT, and BbF) using a photomultiplier as transducer [28], so the optimal chemical parameters have been optimized. Table 1 shows a summary of the optimal chemical parameters for this optosensor, which are the same for the optosensor proposed in this paper.

\subsection{Input entrances}

Seven emission wavelengths were chosen as input values: the four maxima emission wavelengths of the four PAHs and the wavelengths used in the optosensor with a PMT transducer previously developed by [28] (see Fig. 2).

Table 1

Optimized chemical and FIA parameters

\begin{tabular}{ll}
\hline Parameters & Optima value \\
\hline Solid support $(80-120 \mu \mathrm{m})$ & Amberlite XAD 4 \\
Regenerative solution & Acetone \\
Organic solvent in the sample & $25 \%$ of 1,4-dioxane \\
Organic solvent in the carrier solution & None \\
pH & 7.0 \\
Buffer solution and concentration $(15 \mathrm{mM})$ & $\mathrm{H}_{2} \mathrm{PO}_{4}-/ \mathrm{HPO}_{4}{ }^{2-}$ \\
Flow rate $\left(\mathrm{ml} \mathrm{min}{ }^{-1}\right)$ & 2.0 \\
Injection volume $(\mu \mathrm{l})$ & 500 \\
\hline
\end{tabular}

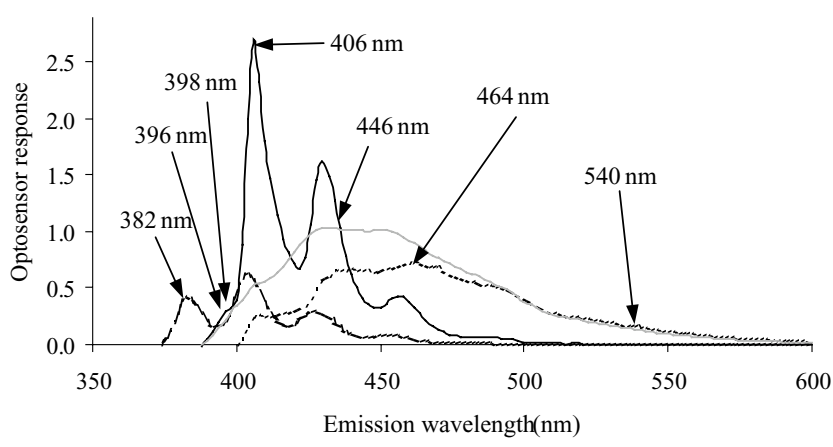

Fig. 2. Emission wavelength set as input values. ANT (----), FLT (….), $\mathrm{BbF}(-)$, and $\mathrm{BaP}(-)$.
Table 2

Optimized parameters used for construction of ANN models

\begin{tabular}{ll}
\hline Parameters & Optima value \\
\hline Input nodes & 7 \\
Hidden nodes & 8 \\
Number of iterations & $>3000$ \\
Output nodes & 4 \\
Learning rate & 0.0005 \\
Weight maximum growth & 1 \\
Weight decay term & 0.0001 \\
\hline
\end{tabular}

\subsection{Artificial neural networks optimization}

There are two important issues to optimize when using ANN's: the architecture and the learning algorithm.

The architecture includes the topology and the kind of nodes. For our purposes we choose an MLP with a single hidden layer, in addition to input and output layers. So the ANN has an input layer with seven nodes $(382,396,398$, 406, 446, 464 and $540 \mathrm{~nm}$ ), an output layer with four nodes (one for each PAH) and a hidden layer. Nodes in the hidden layer will have sigmoid activation function and nodes in the output layer will have linear activation function.

The number of nodes in the hidden layer is an important parameter to optimize. Different architectures of ANN's with a number of the nodes in the hidden layer varying between two and 20 were tested. To compare the different models, the Root of the Mean Square Error (RMSE) was calculated:

RMSE $=\sqrt{\frac{1}{4 n} \sum_{p \in \text { example }} \sum_{j \in \text { output }}\left(c_{p j}-o_{p j}\right)^{2}}$

where $c_{p j}$ and $o_{p j}$ are the predicted and the real concentration for compound $p$ in sample $j$, respectively, $n$ is the number of examples and four is the number of outputs.

Among the extense number of learning algorithms we decided to use QuickProp, because it represents a compromise chosen between the simplicity of vanilla Backprogation and the computational burden of higher methods (e.g. Levenberg-Marquardt). QuickProp usually renders good results (both in terms of convergence rate and learning levels) with a ligther computational complexity. After a number of preliminary experiments we checked that the optimality of the solutions obtained through QuickProp was good enough for our ends. These experiments let us also select the parameteres to used with QuickProp. The selected values are shown on Table 2.

To get robust result a cross-validation procedure has been applied. We used 10 -fold cross validation over the 96 sample data set. This means the data set was randomly partitioned into 10 pieces according to a uniform distribution. This way, 10 different partitions of data into training and test set are available: each of the ten parts was used as a test set and the union of the other parts composed the training set. A given 


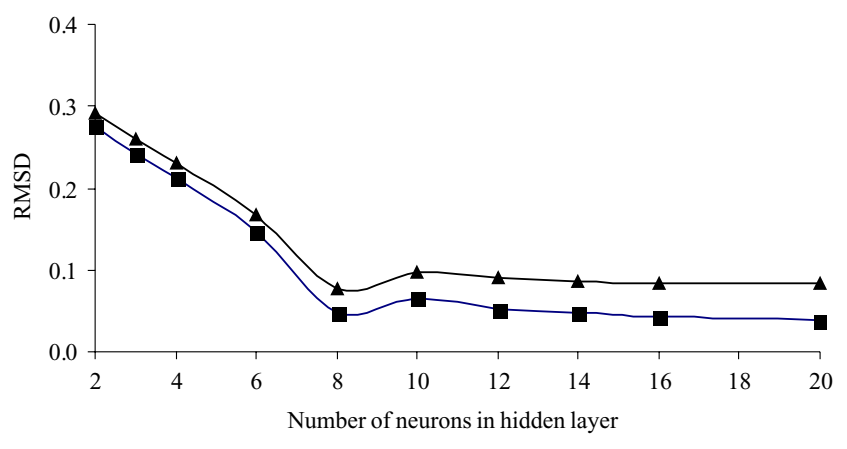

Fig. 3. Plots of RMSE as a function of the number of nodes in hidden layer for $(\mathbf{\square})$ train standards, and $(\boldsymbol{\Delta})$ evaluate standards.

neural net was trained (and evaluated) 10 times on every partition, each one with a different set of initial weights. The final error was taken as the average of the 10 independent executions.

Fig. 3 shows that the lowest RMSE values were obtained employing an eight-node hidden layer. We selected the architecture 7-8-4 because it yielded the best results and it is not very complex. A common principle in Machine Learning is the 'Occam razor' [29], which implies that one should select the simplest system which achieves a desired performance. While networks with more complex architecture display better training performances, they tend to over-fit. This means that they will pay 'too much' attention to specific data of the training set instead of seeking the general trends which would let them give proper answers to previously unseen data.

So the optimal architecture of the neural network used to process the data obtained with the optosensor is 7-8-4 ANN.

\subsection{Effects caused by foreign ions or species}

We made a systematic study of the effects of foreign ions and other PAHs upon the determination of the four
PAHs at $100 \mathrm{ng} \mathrm{ml}^{-1}$. Bicarbonate was tested at a concentration of $1500 \mathrm{mg} \mathrm{l}^{-1}$ and other potentially interfering ions at $150 \mathrm{mg} \mathrm{l}^{-1}$ (five times higher than their usual value in water) [30]. The presence of the usual ions to be found in drinking water caused no interference at the concentrations tested.

The other PAHs, which do not interact with the solid support, were tested at a maximum concentration of $600 \mathrm{ng} \mathrm{ml}^{-1}$ (a ratio of 1:6 compared to the other four PAHs) and caused no interference.

\subsection{Applications of the optosensor}

To test the predictive ability of the present optosensor, drinking water from the city of Granada and two surrounding villages (Atarfe and Gojar) and three-commercial mineral waters (Lanjaron, Zambra, and Fontbella) were spiked with the four PAHs at different levels in the presence of $100 \mathrm{ng} \mathrm{ml}^{-1}$ of each one of the other 12 EPA-PAHs. The waters underwent no previous treatment.

The samples were prepared by adding PAHs combination levels indicated in the Table $3,1 \mathrm{ml}$ of a solution of $25 \times 10^{3} \mathrm{ng} \mathrm{ml}^{-1}$ of each one of the other 12 EPA-PAHs, $25 \%$ (v/v) 1,4-dioxane and $15 \mathrm{mM} \mathrm{H}_{2} \mathrm{PO}_{4}{ }^{-} / \mathrm{HPO}_{4}{ }^{2-}$ buffer solution at $\mathrm{pH} 7.0 .5 \mathrm{ml}$ of sample were injected through valve $\mathrm{A}$ and into a channel of $15 \mathrm{mM} \mathrm{H}_{2} \mathrm{PO}_{4}{ }^{-} / \mathrm{HPO}_{4}{ }^{2-}$ buffer solution at $\mathrm{pH} 7$. Fluorescence emission spectra were reordered at optimal instrumental conditions. The emission spectra were processed to extract the input values and processed by the 7-8-4 ANN trained previously. The results are set out in Table 3.

Table 3 shows that the optosensor proposed can able to detect the presence of ANT, FLT, BaP and/or BbF and quantify the quantity of these PAHs in presence of the other 12 EPA-PAHs in real water samples (tap and mineral water) with adequate relative standard deviations (the European Union and World Health Organization have laid down that

Table 3

Analytical application results in water samples

\begin{tabular}{|c|c|c|c|c|c|c|c|c|c|c|c|c|}
\hline \multirow[t]{2}{*}{ Water sample } & \multicolumn{4}{|c|}{ Added value $\left(\mathrm{ng} \mathrm{ml}^{-1}\right)$} & \multicolumn{4}{|c|}{ Found value $\left(\mathrm{ng} \mathrm{ml}^{-1}\right)$} & \multicolumn{4}{|c|}{ Recovery percentage $(\%)$} \\
\hline & ANT & $\mathrm{BaP}$ & FLT & $\mathrm{BbF}$ & ANT & $\mathrm{BaP}$ & FLT & $\mathrm{BbF}$ & ANT & $\mathrm{BaP}$ & FLT & $\mathrm{BbF}$ \\
\hline \multirow[t]{2}{*}{ Granada city } & - & 100 & - & - & -5.4 & 102.3 & -4.5 & -0.3 & - & 102.3 & - & - \\
\hline & & & & R.S.D. $(\%)$. & 14.5 & 5.9 & 16.7 & 5.6 & - & 5.9 & - & - \\
\hline \multirow[t]{2}{*}{ Atarfe village } & 75 & 75 & 75 & - & 71.5 & 76.4 & 73.2 & 4.7 & 95.3 & 101.8 & 97.6 & - \\
\hline & & & & R.S.D. $(\%)$. & 5.7 & 5.0 & 6.1 & 6.9 & 7.5 & 6.7 & 8.1 & - \\
\hline \multirow[t]{2}{*}{ Gojar village } & 50 & 50 & 100 & 50 & 55.5 & 53.8 & 98.4 & 47.0 & 111.1 & 107.7 & 98.4 & 94.1 \\
\hline & & & & R.S.D. $(\%)$. & 5.6 & 6.1 & 6.0 & 2.4 & 11.2 & 12.3 & 6.0 & 4.8 \\
\hline \multirow[t]{2}{*}{ Lanjaron } & 75 & 75 & - & - & 76.6 & 73.2 & -4.5 & -0.3 & 102.1 & 97.6 & - & - \\
\hline & & & & R.S.D. $(\%)$. & 6.2 & 4.7 & 16.7 & 5.6 & 8.3 & 6.2 & - & - \\
\hline \multirow[t]{2}{*}{ Zambra } & - & 75 & 100 & 75 & -5.6 & 70.4 & 101.4 & 73.0 & - & 93.9 & 101.4 & 97.4 \\
\hline & & & & R.S.D. $(\%)$. & 9.7 & 5.4 & 3.8 & 4.1 & - & 7.2 & 3.8 & 5.5 \\
\hline \multirow[t]{2}{*}{ Fontbella } & 100 & 25 & 75 & 75 & 105.5 & 26.0 & 71.8 & 70.3 & 105.5 & 104.1 & 95.7 & 93.8 \\
\hline & & & & R.S.D. $(\%)$. & 5.6 & 2.9 & 8.2 & 5.0 & 5.6 & 11.7 & 11.0 & 6.6 \\
\hline
\end{tabular}


in assessing the quality of water for human consumption a relative standard deviation of $25 \%$ [31]).

\section{Conclusions}

An optosensor has been developed for determining and quantifying ANT, BaP, FLT, and BbF in water samples in presence of the other 12 EPA-PAHs and without the interference of high-level of ions habitually present in consumption water samples. This optosensor use a photomultiplier coupled with a multilayer perceptron using the QuickProp algorithm for training.

The proposed optosensor is able to measure the four PAHs simultaneously and spending a very short time, for this reason it shows better characteristics than the electrophoretic and/or chromatographic determinations described in the literature. It also displays good characteristics regarding portability, low cost and sensibility, so it presents better conditions than expensive multichannel CCD systems. In conclusion, the optosensor designed is a good tool for using in a real-world environmental.

\section{Acknowledgements}

The authors gratefully acknowledge the financial support of the Spanish Ministerio de Ciencia y Tecnología (Project PPQ2000-1291-C02-01) and a grant from the Consejería de Medio Ambiente de la Junta de Andalucía (Agreement no. 1870). The authors are also grateful to DGICYT for their support to project 30P1379100.

\section{References}

[1] J.F. Fernández Sánchez, A. Segura Carretero, C. Cruces Blanco, A. Fernández Gutiérrez, Anal. Chim. Acta 462 (2002) 217.

[2] J.M. Costa Fernández, A. Sanz Medel, Chapter X: room temperature phosphorescence optical sensors, in: A. Fernández Gutiérrez, S.G. Schulman (Eds.), Analytical Molecular Phosphorimetry: A Practice Approximation, Editorial Universidad de Granada, Granada, 2001 (in Spanish language).

[3] J. Janata, M. Josewick, Anal. Chem. 70 (1998) 179R.
[4] I.V. Tetko, D.J. Livingstone, A.I. Luik, J. Chem. Inform. Compt. Sci. 35 (1995) 826-833.

[5] M. Jalali-Heravi, M.H. Fatemi, Anal. Chim. Acta 415 (2000) 95103.

[6] M. Shamsipur, B. Hemmateenejad, M. Akhond, Anal. Chim. Acta 461 (2002) 147-153.

[7] M. Shamsipur, B. Hemmateenejad, M. Akhond, J. AOAC Int. 85 (2002) 555-562.

[8] I. Kuzmanovski, S. Aleksovska, Chemometr. Intell. Lab. Syst. 67 (2003) 167-174.

[9] A. Hierlemann, U. Weimar, G. Kraus, G. Gauglitz, W. Gopel, Sensor Mater. 7 (1995) 179-189.

[10] A.R. Barron, IEEE Trans. Inform. Theory 39 (1993) 930.

[11] S.E. Fahlman, Faster-learning variations on back-propagation: an empirical study, in: D. Tourezky, G.E. Hinton, T.J. Sejnowski (Eds.), Proceedings of the 1988 Connectionist Models Summer School, Morgan Kaufmann, San Mateo, CA, 1988, pp. 38-51.

[12] S.L. Anker, P.C. Jurs, Anal. Chem. 64 (1992) 1157.

[13] S. Haykin, Neural Network, Prentice-Hall, Englewood Cliffs/New York, 1994.

[14] N.K. Bose, P. Liang, Neural Network, Fundamentals, McGraw-Hill, New York, 1996.

[15] F. Despagne, D.L. Massart, Analyst 123 (1998) 157R.

[16] R. Ferrer, J. Guiteras, J.L. Beltrán, Anal. Chim. Acta 384 (1999) 261.

[17] J. Zupan, J. Gasteiger, Neural Networks in Chemistry and Drugs Design, CVH, Weinheim, 1999.

[18] M.J. Heravi, F. Parastar, J. Chromatogr. A 903 (2000) 145.

[19] M. Jalali-Heravi, Z. Garkani-Nejad, J. Chromatogr. A 927 (2001) 211.

[20] M.H. Fatemi, J. Chromatogr. A 955 (2002) 273.

[21] SNNS, Stuttgart Neural Network Simulator, University of Sttutgart/ University of Tübingen, Germany, 1989-2002, refer to http://wwwra.informatik.uni-tuebingen.de/SNNS/.

[22] J.A. Erustes, A. Andrade-Eiroa, A. Cladrea, R. Forteza, V. Cerdá, Analyst 126 (2001) 451.

[23] H.P. Nirmaier, E. Fisher, A. Meyer, G. Henze, J. Chromatogr. A 730 (1996) 169

[24] G. Michor, J. Carron, S. Bruce, D.A. Cancilla, J. Chromatogr. A 732 (1996) 85.

[25] T.A. Mastran, A.M. Dietrich, D.L. Gallagher, T.J. Grizzard, Water Res. 28 (1994) 2353.

[26] G.A. Junk, J.J. Richard, Anal. Chem 60 (1988) 451.

[27] D.W. Potter, J. Pawliszyn, Environ. Sci. Technol. 28 (1994) 298.

[28] J.F. Fernández Sánchez, A. Segura Carretero, J.M. Costa Fernández, R. Pereiro, C. Cruces Blanco, A. Sanz Medel, A. Fernández Gutiérrez, Anal. Bioanal. Chem 377 (2003) 614-623.

[29] C.M. Bishop, Neural Networks for Pattern Recognition, Cambridge University Press, 1995.

[30] J. Catalán Lafuente, Química del agua, Librería Editorial Bellisco, Madrid, 1981.

[31] European Directive 98/83/EC. 